\title{
Theme: Pediatric Nephrology
}

\section{8}

Clinical outcomes in children with Henoch-Schönlein purpura nephritis without crescents. (Pediatr Nephrol. 2017 Feb 15. doi: 10.1007/s00467-017-3604-9. [Epub ahead of print])

Henoch-Schönlein purpura(HSP) is the most common vasculitis in children. Its long-term prognosis depends on renal involvement. The management of HSP nephritis (HSPN) remains controversial. This study reports the prognosis of children with HSP presenting with class-2 ISKDC (International Study of Kidney Disease in Children) nephritis. All such children diagnosed between 1995 and 2015 in four pediatric nephrology centers were included, and clinical and biological data were collected from the medical files. The primary endpoint was remission of proteinuria defined as $<200 \mathrm{mg} / \mathrm{L}$. Ninety-two children were included in the study with a median (range) follow-up of 36 (6-120) months; $28 \%$ had nephrotic syndrome, $31 \%$ had proteinuria $>3 \mathrm{~g} / \mathrm{L}, 52 \%$ had proteinuria $1-3 \mathrm{~g} /$ $\mathrm{L}$, and $18 \%$ proteinuria $<1 \mathrm{~g} / \mathrm{L}$. Forty-seven percent of patients received treatment with oral steroids alone, and $37 \%$ received methylprednisolone pulses followed by oral steroids, and $18 \%$ did not receive steroids. Although $85 \%$ reached remission during followup, $12 \%$ did not maintain complete remission over time so that only $75 \%$ remained in complete remission by the end of the follow-up. Univariate analysis found a higher likelihood of remission in patients with higher proteinuria at disease onset $(P=0.009)$. This trend was not found in the multivariate analysis after adjusting for treatments, as patients with higher proteinuria were most often treated with steroids. This study concludes that one-fourth of patients with HSPN class-2 remain proteinuric, and thus carry the risk of developing chronic kidney disease over the long term. This finding, together with the better outcome of patients treated with steroids, is in favor of using high-dose oral or intravenous steroids in these patients.

\section{Incidence and outcome of acute cardiorenal syndrome in hospitalized children. (Indian J Pediatr. $2017 \mathrm{Feb}$ 27. doi: 10.1007/s12098-017-2307-3. [Epub ahead of print])}

The objective of this prospective cohort study was to determine the incidence, etiology and outcome of Cardiorenal syndrome (CRS) in 242 children (age 6 mo-18 y) hospitalized with primary cardiac, renal or any systemic disorder at a tertiary care center in India. Sixtyseven (27.7\%) children developed CRS; $40.3 \%, 20.9 \%$, and $38.8 \%$ had CRS-1, 3 and 5, respectively. Cardiac diseases leading to CRS were myocarditis $(40.7 \%)$ followed by congenital heart disease $(25.9 \%)$, rheumatic heart disease $(18.5 \%)$, and dilated cardiomyopathy (7.4\%); renal disease associated with CRS was acute glomerulonephritis (100\%), and major systemic disorders leading to CRS were septicemia (53.8\%), malaria (23.1\%), scrub typhus (7.7\%) and acute gastroenteritis (3.8\%). The occurrence of CRS was associated with an increased risk of mortality (OR 6.3, $95 \%$ CI $2.8,14.1 ; P<0.001)$. A subgroup analysis revealed that children with CRS having acute kidney injury stage 2 and 3 also had a higher risk of mortality. The study concludes that the incidence of CRS is quite high in children with cardiac, renal or systemic diseases, and is associated with a significant risk of mortality. Children presenting with these illnesses should be monitored for the occurrence of CRS so that early intervention may reduce mortality.

\section{Outcome of extremely low birth weight infants with a history of neonatal acute kidney injury. (Pediatr Nephrol. 2017 Feb 14. doi: 10.1007/s00467-017-3582-y. [Epub ahead of print])}

The objective of this study was to evaluate the outcome of extremely low birth weight (ELBW) infants with a history of acute kidney injury (AKI). Medical records of all ELBW infants admitted to the neonatal intensive care unit (NICU) between Jan 2002 and Dec 2011 were reviewed for infants' demographics, blood pressure (BP) at NICU discharge and at $\geq 3$ years, and estimated glomerular filtration rate (eGFR) at $\geq 2$ years. Out of 222 included patients, $10 \%$ had AKI stage 2 and 3,39\% had AKI stage 1 , and the rest did not have AKI. At NICU discharge, there was a difference in diastolic $\mathrm{BP}$ (DBP) among infants who had AKI stages 2 and 3, those who had stage 1 , and those who did not have AKI, and $11 \%$ had hypertension (HTN). Although there was a significant correlation between the rise in $\mathrm{SCr}$ and DBP at NICU discharge in infants with $\mathrm{AKI}(\mathrm{R}=0.304 ; P=0.004)$, there was no difference in $\mathrm{HTN}$ between infants with and those without AKI. At $\geq 2$ years of age, five children across all groups had an eGFR $<90 \mathrm{ml} / \mathrm{min} / 1.73 \mathrm{~m}^{2}$ or chronic kidney disease (CKD). At $\geq 3$ years of age, $5 \%$ (11 out of 222) had HTN. The study concluded that at NICU discharge, infants with AKI stages 2 and 3 have a higher DBP than infants with stage 1 AKI and those who did not have AKI. However, there is no difference in the rate of HTN between the two groups. At $\geq 2$ years ELBW infants are at risk for CKD independently of whether or not they develop neonatal AKI.

\section{Association between vancomycin trough concentrations and acute kidney injury in neonates. (BMC Pediatr. 2017 Feb 11;17(1):50. doi: 10.1186/s12887-017-0777-0)}

The objective of this study was to compare the incidence of acute kidney injury (AKI) in neonates with serum vancomycin trough concentrations $<10 \mathrm{mg} / \mathrm{L}, 10-15 \mathrm{mg} / \mathrm{L}$, or $>15 \mathrm{mg} / \mathrm{L}$. Aretrospective chart review of patients in the neonatal intensive care unit (NICU) was conducted to determine the incidence of AKI in neonates receiving vancomycin. The overall incidence of AKI was $2.7 \%$. Comparison of the incidence of AKI in the three groups showed a statistically significant association between increasing vancomycin trough concentration and incidence of AKI. The study concluded that there is low incidence of AKI in neonates receiving vancomycin. However, there is a positive correlation between increasing vancomycin trough concentrations and an increasing serum creatinine.

SRIRAM KRISHNAMURTHY drsriramk@yahoo.com 